\section{Inflammatory seborrheic keratosis resolution after hyperbaric oxygen therapy: Case presentation and pathophysiology review}

Karin Elman-Shina, ${ }^{1}$ Monica Elman, ${ }^{2}$ Shai Efrati ${ }^{1,3,4}$

${ }^{1}$ Sagol Center for Hyperbaric Medicine and Research, Shamir (Assaf Harofeh) Medical Center, Zerifin; ${ }^{2}$ Elman Dermatology and Laser Clinics, Tel Aviv; ${ }^{3}$ Sackler School of Medicine, TelAviv University, Tel-Aviv; ${ }^{4}$ Sagol School of Neuroscience, Tel-Aviv University, Tel-Aviv, Israel

\begin{abstract}
Seborrheic keratosis (SK) is a common epidermal tumor, consisting of a benign proliferation of immature keratinocytes. The natural history of SK is a slow progression over time and complete remission is not expected. The article presents the first case of a complete resolution of a large (2.5 $\mathrm{cm}$ diameter) SK lesion after hyperbaric oxygen therapy (HBOT). In addition to the case presentation, the pathophysiology of SK and the potential beneficial physiological effects of HBOT are reviewed and discussed.
\end{abstract}

\section{Introduction}

Seborrheic keratosis (SK) is a common epidermal tumor, consisting of a benign proliferation of immature keratinocytes. Clinically, SK manifests as solitary or multiple, well demarcated brownish papules or plaques with a verrucous surface, that predominantly localizes at the head, neck and trunk areas. ${ }^{1}$ Since the tumor slowly grows, in many cases the lesion is surgically removed due to cosmetic reasons or because the lesions are traumatized and become symptomatic. ${ }^{2-5}$

In this article we report an unexpected full resolution of a large SK lesion in a patient treated by hyperbaric oxygen therapy (HBOT) due to hemorrhagic cystitis. In addition to the case presentation, the pathophysiology of SK and the potential beneficial physiological effects of HBOT in the setting of SK will be reviewed and discussed.

\section{Case Report}

A 74 years old man was admitted for HBOT due to chronic late post radiation hemorrhagic cystitis (grade 3-4 RTOG). In addition, on his left temple, he had a slowly growing SK lesion, known for about 24 months prior to the treatment. The SK presented as a red non-tender demarcated lesion, $2.5 \mathrm{~cm}$ diameter in size, with clear boundaries and occasional mild oozing (Figure 1A).

The lesion was diagnosed as inflammatory seborrheic keratosis and was scheduled for surgical removal after the intendent HBOT.

The medical history of the patient included prostate cancer (Glisson score 6), diagnosed 13 years prior to his admission, that was treated with radiation. Since he had chronic unremitting hemorrhagic cystitis, he was referred to HBOT. In addition to the cystitis, he had type-II diabetes mellitus, hypertension, osteoporosis, gastroesophageal reflux, hyperlipidemia, s/p resection of craniopharyngioma in 2019 and a fractionated stereotactic radiation therapy, gait disturbance with parkinsonism and a primary unspecified kidney tumor.

The HBOT protocol used for his hemorrhagic cystitis included a total of 56 hyperbaric sessions, five days per week of 90 minutes $100 \%$ oxygen at 2 ATA with fiveminute air breaks every 20 minutes. The treatment went well with no significant side effects and the clinical symptoms of the hemorrhagic cystitis resolved.

Surprisingly, in addition to the resolution of the cystitis, there was a full resolution of the $2.5 \mathrm{~cm}$ seborrheic keratosis lesion (Figure 1B). During a follow-up, 16 weeks after the last hyperbaric session, the skin was still intact without any sign of recurrence.

\section{Discussion and Conclusions}

SKs are common epidermal tumors that usually develop after the age of 50. Skin aging and cumulative UV exposure are considered to play a major role in SK pathogenesis and seem to cause increased expression of amyloid precursor protein (APP), ${ }^{6}$ which is a marker of cellular senescence and chronic inflammation, particularly in human keratinocytes (Figure 2 summarizes the pathophysiology cascade of SK). ${ }^{7}$

APP expression has been evaluated in SKs versus normal skin by different methods and it was found that APP and its downstream products (i.e. amyloid- $\beta 42$ ) are highly expressed in SK lesions as compared to
Correspondence: Karin Elman Shina, Shamir Sagol Center for Hyperbaric Medicine and Research, Shamir (Assaf Harofeh) Medical Center, Zerifin, 70300, Israel.

E-mail: karine@shamir.gov.il

Key words: Inflammatory, seborrheic keratosis, SK, hyperbaric.

Contributions: KES \& SE wrote the first draft of the article and treated the patient presented. ME reviewed and worked on the final version of the manuscript.

Conflict of interest: The authors declare no potential conflict of interest.

Funding: None.

Ethics approval: Not relevant as a case report and review of the literature.

Consent to publication: The patient gave his consent to publish his case.

Availability of data and materials: Data can be available on request at the Sagol center for Hyperbaric Medicine, Shamir medical center, Israel.

Please cite this article as: Elman-Shina K, Elman M, Efrati S. Inflammatory seborrheic keratosis resolution after hyperbaric oxygen therapy: Case presentation and pathophysiology review. Dermatol Rep 2021;13:8871.

Received for publication: 30 August 2020. Accepted for publication: 2 October 2020.

This work is licensed under a Creative Commons Attribution-NonCommercial 4.0 International License (CC BY-NC 4.0).

C Copyright: the Author(s), 2021

Licensee PAGEPress, Italy

Dermatology Reports 2021; 13:8871

doi:10.4081/dr.2021.8871

the adjacent normal skin tissues. ${ }^{8}$ Similar to growth factors and tumor growth factor alpha (TGF- $\alpha$ ), APP can induce proliferation of epidermal keratinocytes, and contribute to mitochondrial dysfunction and oxidative phosphorylation. ${ }^{7}$ In addition to the skin, APP is highly expressed and has a pathophysiology role in many of the agerelated diseases such as Alzheimer's disease, atherosclerosis, Parkinson and macular degeneration. ${ }^{9-11}$ HBOT utilizes $100 \%$ oxygen in an environmental pressure higher than one absolute atmosphere (ATA) to enhance the amount of oxygen dissolved in body's tissues. Repeated intermittent hyperoxic exposures have been shown to induce physiological effects which normally occur during hypoxia in a hyperoxic environment, 
including stem cells proliferation, generation of new blood vessels (angiogenesis) and enhanced tissue regeneration. ${ }^{12-16}$ The direct effect of HBOT on keratinocytes was evaluated by the use of monolayer cultures including dermal fibroblasts, keratinocytes and melanocytes. ${ }^{17}$ It was found that repeated HBOT sessions at a pressure of 2 atmospheres, as was used in our treatment protocol, inhibits keratinocytes proliferation. ${ }^{17} \mathrm{In}$ addition, in another study it was demonstrated that HBOT ameliorates APP and amyloid beta $(A \beta)$ plaques in the brain of Alzheimer prone mice. ${ }^{18}$ With regards to UV related injury, it was found that pretreatment with HBOT significantly reduces UV-A induced apoptosis and proliferation in hairless SKH1-E mice. ${ }^{19}$ Hence, as summarized in Figure 2, HBOT has the poten-
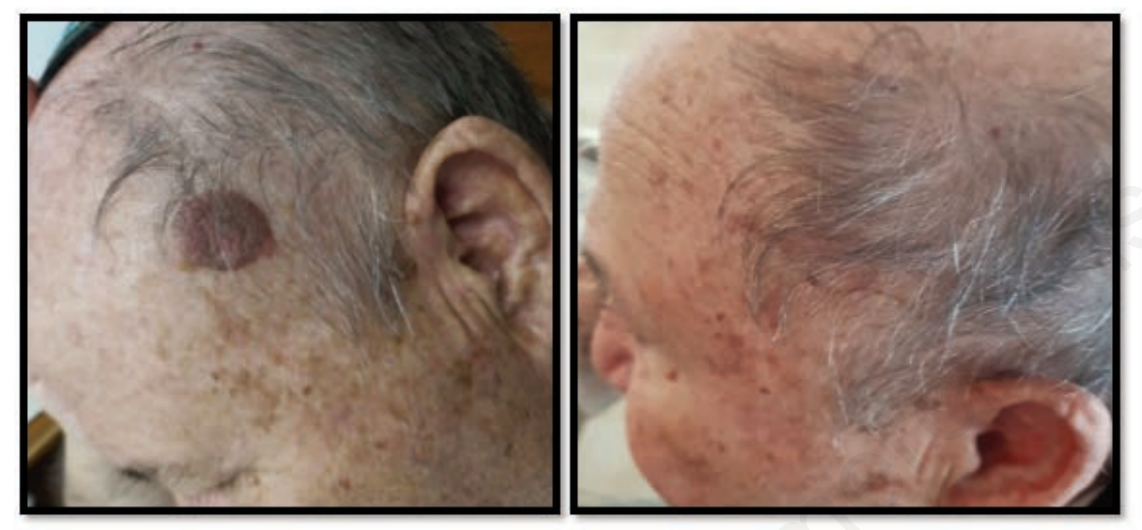

Figure 1. The skin before and after hyperbaric oxygen therpy. A) before treatment; B) after treatment.

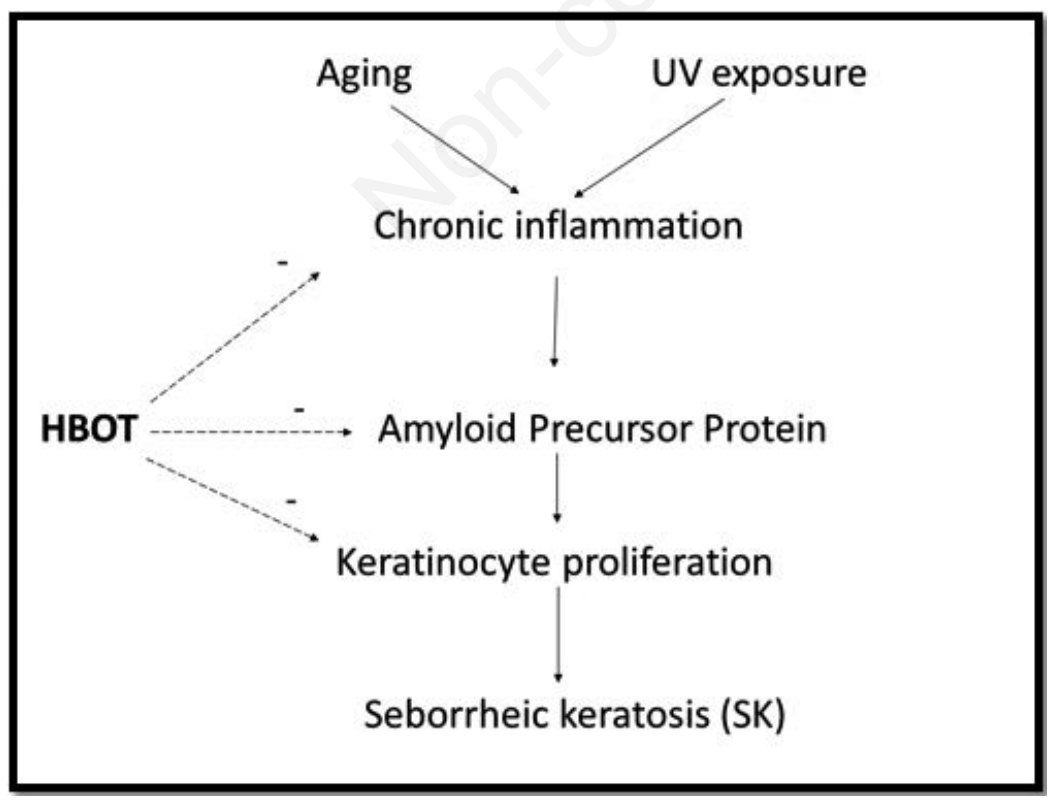

Figure 2. The pathophysiology cascade of seborrheic keratosis and the biological effect of hyperbaric oxygen therapy. tial to intervene and revert the pathophysiological processes responsible for the development of SK lesions.

In our patient, the SK lesion at a diameter of $2.5 \mathrm{~cm}$ had a complete remission after HBOT (Figure 1).

The natural history of SK is a slow progression over time and complete remission is not expected. The only available treatments for SK include surgical removal, cryosurgery, curettage, electrodessication, shave excision or laser therapies (CO2, YAG). ${ }^{3-5}$ To the best of our knowledge, this is the first reported case of complete resolution of SK without local intervention. Had the lesion not been so large in size and not at such a notable location, we would have probably missed HBOT's effect on the lesion. Until this case, we have not moni-

\section{References}

1. Hafner C, Vogt T. Seborrheic keratosis. J Dtsch Dermatol Ges 2008;6:664-77.

2. Schlesinger TE, Favre C. Enhancing Outcomes in Seborrheic Keratosis: Using a Novel Treatment Solution. J Drugs Dermatol 2019;18:178-82.

3. Culbertson GR. 532-nm diode laser treatment of seborrheic keratoses with color enhancement. Dermatol Surg 2008;34:525-8.

4. Kim YK, Kim DY, Lee SJ, et al. Therapeutic efficacy of long-pulsed 755-nm alexandrite laser for seborrheic keratoses. J Eur Acad Dermatol Venereol 2014;28:1007-11.

5. Polder KD, Landau JM, Vergilis-Kalner IJ, et al. Laser eradication of pigmented lesions: a review. Dermatol Surg 2011;37:572-95.

6. Cheong KA, Lee AY. Guanine Deaminase Stimulates Ultravioletinduced Keratinocyte Senescence in Seborrhoeic Keratosis via Guanine Metabolites. Acta Derm Venereol, 2020.

7. Li Y, Wang Y, Zhang W, et al. Overexpression of Amyloid Precursor Protein Promotes the Onset of Seborrhoeic Keratosis and is Related to Skin Ageing. Acta Derm Venereol Acta Derm Venereol 2018;98:594-600.

8. Wollina U. Seborrheic Keratoses - The Most Common Benign Skin Tumor of Humans. Clinical presentation and an update on pathogenesis and treatment options. Open Access Maced J Med Sci 2018;6:2270-5.

9. Tibolla G, Norata GD, Meda C, et al. Increased atherosclerosis and vascular inflammation in APP transgenic mice with apolipoprotein E deficiency. Atherosclerosis 2010;210:78-87.

10. Schulte, E.C., et al., Rare variants in beta-Amyloid precursor protein (APP) and Parkinson's disease. Eur J Hum Genet 2015;23:1328-33.

11. Yoshida T, Fukumori A, Mollenhauer B, 
et al. The potential role of amyloid beta in the pathogenesis of age-related macular degeneration. $\mathrm{J}$ Clin Invest 2005;115:2793-800.

12. Cimino F, Balestra C, Germonpré $P$, et al. Pulsed high oxygen induces a hypoxic-like response in human umbilical endothelial cells and in humans. J Appl Physiol (1985) 2012;113:1684-9.

13. Sunkari VG, Lind F, Botusan IR, et al. Hyperbaric oxygen therapy activates hypoxia-inducible factor 1 (HIF-1), which contributes to improved wound healing in diabetic mice. Wound Repair Regen 2015;23:98-103.
14. Milovanova TN, Bhopale VM, Sorokina EM, et al. Hyperbaric oxygen stimulates vasculogenic stem cell growth and differentiation in vivo. $\mathrm{J}$ Appl Physiol (1985) 2009;106:711-28.

15. Yang $Y$, Wei $H$, Zhou $X$, et al. Hyperbaric oxygen promotes neural stem cell proliferation by activating vascular endothelial growth factor/extracellular signal-regulated kinase signaling after traumatic brain injury. Neuroreport 2017;28:1232-8.

16. Hadanny A, Efrati S. The HyperoxicHypoxic Paradox. Biomolecules 2020;10:6.
17. Dimitrijevich SD, Paranjape S, Wilson JR, et al. Effect of hyperbaric oxygen on human skin cells in culture and in human dermal and skin equivalents. Wound Repair Regen 1999;7:53-64.

18. Shapira R, Efrati S, Ashery U. Hyperbaric oxygen therapy as a new treatment approach for Alzheimer's disease. Neural Regen Res 2018;13:817-8.

19. Fuller AM, Giardina C, Hightower LE, et al. Hyperbaric oxygen preconditioning protects skin from UV-A damage. Cell Stress Chaperones 2013;18:97107. 